\title{
Attentional control and reflexive orienting to gaze and arrow cues
}

\author{
JELENA RISTIC \\ University of California, Santa Barbara, California \\ Alissa Wright \\ University of Toronto, Toronto, Ontario, Canada \\ AND \\ Alan Kingstone \\ University of British Columbia, Vancouver, British Columbia, Canada
}

\begin{abstract}
A wealth of data indicate that central spatially nonpredictive eyes and arrows trigger very similar reflexive spatial orienting, although the effects of eyes may be more strongly reflexive (e.g., Friesen, Ristic, \& Kingstone, 2004). Pratt and Hommel (2003) recently reported that the orienting effect for arrows is sensitive to arbitrary cue-target color contingencies; for example, an attentional orienting effect for blue colored arrows is evident only for blue targets. We reasoned that if the orienting effect elicited by eye direction is more strongly reflexive than the orienting effect elicited by arrow direction, it follows that eyes, unlike arrows, may trigger orienting effects that generalize across congruent and incongruent cue-target color contingencies. Replicating Pratt and Hommel (2003), we found that the reflexive attention effect elicited by arrows is specific to color-congruent target stimuli. The attention effect triggered by eyes, however, generalizes across color-congruent and color-incongruent target stimuli. These data support the hypothesis that eye direction and arrow direction trigger similar reflexive shifts in spatial attention, but that the attention effect triggered by eye direction is more strongly reflexive.
\end{abstract}

In everyday life human eyes are a vital nonverbal social cue that enables fluent social communication between individuals. Transmission of gaze information is facilitated by a uniquely human eye morphology, with its dark iris surrounded by a high contrast white sclera, which allows people to accurately convey to others where they are looking (Kobayashi \& Koshima, 1997). Perception of gaze information also seems to be supported by specific neural architecture - the superior temporal sulcus (STS; Allison, Puce, \& McCarthy, 2000) — with evidence of its engagement emerging within days of birth (see, e.g., Batki, Baron-Cohen, Wheelwright, Connellan, \& Ahluwalia, 2000).

Researchers have developed a simple task to measure the tendency for children and adults to attend to where someone else is looking (Friesen \& Kingstone, 1998; Hood, Willen, \& Driver 1998). Friesen and Kingstone asked participants to detect, localize, and identify visual targets that appeared to the left or right of a central face on a computer monitor. The key manipulation was that just before a target appeared, the eyes in the face on the computer screen would look to the left or right of center. Friesen and Kingstone found that the response time (RT) to a visual target was shortest when it appeared at the gazed-at location. This RT advantage for a target at the cued (gazed-at) location occurred despite the fact that the participants knew in advance that the eyes in the face did not predict where a target would occur. Based on these data, and the fact that the RT advantage at the cued location emerged very rapidly, Friesen and Kingstone suggested that attentional orienting to gaze direction is reflexive and as such may engage brain processes that are especially tuned for gaze direction (Allison et al., 2000; Langton, Watt, \& Bruce, 2000).

Subsequent to these original investigations, a body of evidence emerged showing clearly that other directional cues such as arrows and words produce a reflexive attention effect that is comparable to the attention effects produced by eyes (Hommel, Pratt, Colzato, \& Godijn, 2001; Tipples, 2002). Together, these findings question the original idea that orienting to eye gaze may be unique.

Friesen, Ristic, and Kingstone (2004) addressed this issue directly by testing whether arrows trigger attention shifts that are as strongly reflexive as eyes. They compared performance elicited by eyes and arrows when each of these cues was counterpredictive; for example, if a participant saw eyes or arrows indicating a left location, the target was highly likely to occur at the opposite, right loca-

J. Ristic, ristic@psych.ucsb.edu 
tion. Friesen et al. found that only eyes triggered an initial, rapid reflexive shift in attention to the cued location. In other words, participants were unable to avoid attending reflexively to where the eyes were looking, but they were able to avoid attending reflexively to where arrows were pointing. This finding - that eyes are more strongly reflexive than arrows - lends support to the idea that orienting to eye direction is strongly reflexive as it is relatively resistant to cognitive control.

Considered as a whole, it appears that there is a wide range of central spatial nonpredictive stimuli that trigger reflexive shifts in spatial attention. Some cues, like gaze direction, trigger rapid reflexive shifts of attention that are strongly reflexive and hence they are relatively insensitive to top-down inhibitory control (Friesen et al., 2004; Ristic $\&$ Kingstone, 2005). At the other extreme are stimuli like numbers, which can produce shifts of attention when they are spatially nonpredictive (Fischer, Castel, Dodd, \& Pratt, 2003), but this orienting effect is both slow to emerge and extremely sensitive to changes in the top-down mental set adopted by a participant (Galfano, Rusconi, \& Umiltà, 2006; Ristic, Wright, \& Kingstone, 2006). Arrow stimuli would appear to fall somewhere in the middle, producing rapid shifts of attention even when they are known to be spatially nonpredictive, but their effects are relatively amenable to top-down control.

A recent study by Pratt and Hommel (2003) provides excellent evidence of arrow cues' sensitivity to cognitive control. Participants were presented with a color precue that defined the response target; for example, if the color precue was a blue patch, then participants were to press a key if the target was also colored blue, and to refrain from responding altogether if the target was not colored blue. Prior to a target appearing, four arrows appeared at central fixation, each colored differently and each pointing in different direction (left, right, up, down). Pratt and Hommel found that when participants are set to respond to a target stimulus of a particular color, a task irrelevant arrow stimulus that shares the target color will trigger a shift in spatial attention. That is, if the set is for a target that is colored blue, attention is shifted to the location cued by a blue arrow; and if the set is for a target that is colored green, attention is shifted to the location cued by a green arrow. Importantly, this spatial color-target contingency effect occurred despite the fact that the location of the target was not predicted by the color or direction of the color-congruent arrow.

In the present study, we asked whether these arbitrary cue-target congruency effects found for arrows will also occur for eyes. Based on our hypothesis that the attention effects for arrows are more amenable to arbitrary top-down associations than eyes, the strong prediction is that arrows will be more sensitive to cue-target color contingencies than eyes. Thus, we predict that arrows will produce cuing effects that are specific to congruent cuetarget color contingencies (Pratt \& Hommel, 2003). In contrast, if eyes are more strongly reflexive than arrows, a gaze effect may extend across congruent and incongruent cue-target color contingencies.
We addressed this question using a four location cuing task, modeled on the design used by Pratt and Hommel (2003). The aim of our study was to examine potential differences that cue-target color compatibility may have on attentional orienting triggered by arrow and eye direction. All participants were asked to press one key if the target was white and another key if the target was black. Prior to target onset, a spatially irrelevant arrow cue, or spatially irrelevant schematic eye direction cue, colored white or black appeared at central fixation and was directed left or right. The color and direction of the arrow and eye cue did not predict the color or location of the target. The key question was whether a reflexive spatial effect of the arrow cue would be greater for a congruently colored target compared to an incongruently colored target, and whether the reflexive attention effect that is elicited by eye direction would similarly be affected by cue-target color contingencies.

\section{METHOD}

\section{Participants}

Eighty naive participants were assigned randomly to four equal groups of twenty: a white arrow group, a black arrow group, a black eyes group, and a white eyes group.

\section{Apparatus and Stimuli}

All stimuli were black and white line drawings presented on a gray background. The stimuli were shown on a PowerPC Macintosh computer connected to a 15 -in. color monitor set to black and white. The central arrow cue was a triangle measuring $1^{\circ}$ of visual angle at its base and $0.7^{\circ}$ in height. The schematic eyes, measured $2.6^{\circ}$ and were constructed by combining a circle outline, with an inner filled-in circle representing the pupil. The target $\left(1^{\circ}\right.$ in size) was square- or diamond-shaped. Target shape was varied in case in a future study we wished to ask participants to discriminate the target stimulus on a dimension that was not contained by the cue. The arrow and eye cues were presented at the center of the screen and targets appeared peripherally $3^{\circ}$ away from center fixation. Response keys were " $\mathrm{z}$ " and "/" on a keyboard, with the left index finger pressing the " $z$ " key and the right index finger pressing the "/" key. Color assignment to the response keys was counterbalanced between participants.

\section{Design}

The stimuli and sample sequence of events are illustrated in Figure 1. All participants performed a color discrimination task, pressing one key if the target was black and the other key if the target was white. Each participant completed 480 trials.

For all groups, the central cue indicated one of the two possible directions (left or right), and the target could appear either on the left, right, up, or down. This created two different cue validity conditions: cued trials, in which the target occurred either on the left or right side as indicated by the cue $(p=.25)$; and uncued trials in which the target occurred at the location that was not indicated by the cue. Note that RTs for vertical and horizontal uncued locations will be collapsed in the Results section because an ANOVA revealed that these locations are equivalent $(F<1) .{ }^{1}$ Cue direction, target location, and target features (color and shape) were selected randomly and presented with equal probability. RT to press a key was measured in milliseconds and was timed from target onset.

\section{Procedure}

All trials began with a $105-\mathrm{msec}$ presentation of a central arrow cue or schematic eye cue pointing left or right. Following a 315msec stimulus onset interval (SOA; selected as it has been shown in past studies to produce a large reflexive attention effect for both eyes 


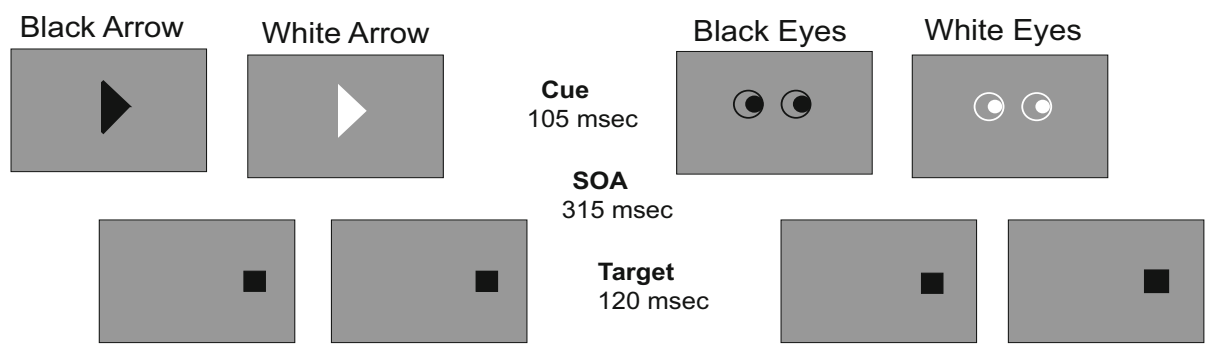

Figure 1. Illustration of stimuli and sample sequence of events. The start of each trial was signaled by presentation of the central cue (black arrow, white arrow, black eyes, or white eyes) pointing left or right for 105 msec. After an SOA of $315 \mathrm{msec}$, a color-congruent or -incongruent target appeared either at the location indicated by the cue or at an uncued location for 120 msec. Participants were asked to report the color of the target by pressing either the " $\mathrm{z}$ " or "/" key on the keyboard. All trials terminated on response or after 1,005 msec, whichever occurred first. The intertrial interval was 2,505 msec. The stimuli are not drawn to scale.

and arrows) a target appeared left, right, above, or below the cue for $120 \mathrm{msec}$. The trial was terminated on response or after $1,005 \mathrm{msec}$, whichever occurred first. The intertrial interval was $2,505 \mathrm{msec}$.

Participants were seated centered in front of the computer screen at an approximate distance of $57 \mathrm{~cm}$. It was emphasized that the direction of the arrow and eyes and their color did not predict location, color, or shape of the target. All participants were asked to respond as quickly and as accurately as possible and to maintain central fixation throughout the experiment.

\section{RESULTS}

Anticipations ( $\mathrm{RT}<100 \mathrm{msec}$ ), timed-out responses (RT $>1,000 \mathrm{msec}$ ), and incorrect keypresses were classified as errors and were excluded from the analysis (see Table 1). Importantly, since the analyses were conducted on correct RTs, in no condition were the RT data contradicted by a speed-accuracy trade-off - that is, faster, yet more errorprone, responses at cued versus uncued locations.

\section{Omnibus Analysis}

Figure 2 presents the mean RTs as a function of cue type (arrow and eyes), cue-target color congruency (e.g., black arrow-black target), and cue spatial validity (cued and uncued locations). As one can see, whenever the cue is black, RT for black targets is facilitated; and whenever the cue is white, RT for white targets is facilitated. This cue-target color congruency effect holds whether or not the cue is an arrow or an eye. Importantly, however, there appears to be a variation in the magnitude of the spatial validity effect (cued vs. uncued locations) as a function of cue type and cue-target color congruency. That is, for black arrow cues a spatial validity effect is present only for black targets, and for white arrow cues a spatial validity effect is present only for white targets. In contrast, for eye cues a spatial validity effect is present for all targets regardless of whether their color is congruent or incongruent with the color of the central eye cues. To test the robustness of these observations, we committed the data to an omnibus ANOVA with cue type, cue-target color congruency, and cue validity as factors. This analysis revealed that the highest order three-way cue type $\times$ congruency $X$ validity interaction was highly significant $[F(3,76)=4.8$, $p<.005]$. The main effect of cue validity $[F(1,76)=$ $17.3, p<.0001]$ and the interaction between cue type and cue-target color congruency were also highly reliable $[F(3,76)=25.6, p<.0001]$. To confirm that the source of the three-way interaction is due to our interpretation above, we analyzed each of the cue types separately.

\section{Arrow Cues}

As suggested by the omnibus ANOVA, the spatial attention effect (cued RT < uncued RT) is greater for color

Table 1

Mean Response Times (in Milliseconds), Standard Deviations, and Percent Error for Each Cue Type $\times$ Cue-Target Congruency $\times$ Cue Spatial Validity Condition

\begin{tabular}{|c|c|c|c|c|c|c|c|c|c|c|c|c|}
\hline & \multicolumn{12}{|c|}{ Cue Type } \\
\hline & \multicolumn{3}{|c|}{ Black Arrow } & \multicolumn{3}{|c|}{ White Arrow } & \multicolumn{3}{|c|}{ Black Eyes } & \multicolumn{3}{|c|}{ White Eyes } \\
\hline & $M$ & $S D$ & $\% \mathrm{E}$ & $M$ & $S D$ & $\% \mathrm{E}$ & $M$ & $S D$ & $\% \mathrm{E}$ & $M$ & $S D$ & $\% \mathrm{E}$ \\
\hline \multicolumn{13}{|l|}{ Congruent } \\
\hline Cued & 407 & 58 & 4.1 & 426 & 72 & 3.6 & 392 & 59 & 4.9 & 398 & 31 & 2.5 \\
\hline H-uncued & 419 & 65 & 4.4 & 435 & 69 & 5.6 & 400 & 57 & 4.7 & 411 & 34 & 2.6 \\
\hline V-uncued & 414 & 60 & 4.1 & 430 & 66 & 5.3 & 397 & 59 & 4.5 & 404 & 31 & 2.7 \\
\hline \multicolumn{13}{|l|}{ Incongruent } \\
\hline Cued & 442 & 49 & 6.7 & 459 & 70 & 5.9 & 413 & 54 & 6.2 & 419 & 41 & 3.6 \\
\hline H-uncued & 439 & 47 & 5.7 & 455 & 62 & 6.4 & 418 & 52 & 6.1 & 423 & 39 & 3.7 \\
\hline V-uncued & 440 & 45 & 4.7 & 461 & 68 & 6.2 & 413 & 54 & 7.0 & 421 & 39 & 3.1 \\
\hline
\end{tabular}

Note-Target locations were either cued, horizontal uncued, or vertical uncued. 


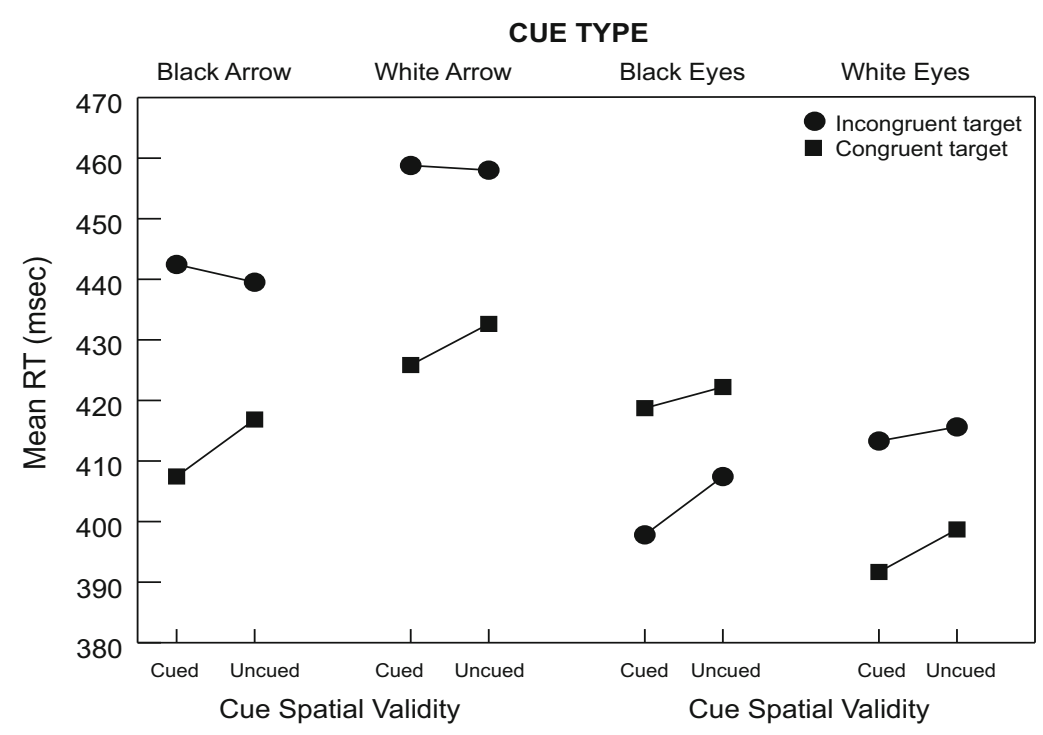

Figure 2. Mean correct response times (RTs) as a function of cue type, cuetarget color congruency, and cue spatial validity.

congruent targets than for color-incongruent targets. For the black arrow group participants identified black targets faster when they appeared at the cued versus the noncued locations. Similarly, for the white arrow group participants identified white targets faster when they appeared at the cued versus the noncued locations. Separate ANOVAs for each group confirmed these observations, with cue spatial validity (cued/uncued) and cue-target color congruency (congruent/incongruent) interacting [black arrow, $F(1,19)=4.7, p<.05$; white arrow, $F(1,19)=$ $4.8, p<.05]$. The main effects of target color were also reliable [black arrow, $F(1,19)=24.6, p<.0001$; white arrow, $F(1,19)=27.0, p<.0001]$, indicating that, for each group, RT is faster overall for the target color that is congruent with the color of the arrow cue. No other effects were significant (all $F \mathrm{~s}<2.4$, all $p \mathrm{~s}>.1$ ).

\section{Eye Cues}

In contrast to arrow cues, the spatial attention effect (cued RT $<$ uncued RT) is present for both color congruent and color incongruent targets. Mirroring the analysis performed with arrow cues, the within-subjects ANOVAs returned significant main effects of cue spatial validity [black eyes, $F(1,19)=4.8, p<.05$; white eyes, $F(1,19)=$ $19.4, p<.0001]$, which, unlike the effects of arrow cues, never interacted with cue-target color congruency (all $F \mathrm{~s}<2.2$, all $p \mathrm{~s}>$.1). Note that cue spatial validity did not interact with cue-target color congruency even though a main effect of cue-target color congruency was significant for both conditions [black eyes, $F(1,19)=14.0, p<$ .01 ; white eyes, $F(1,19)=10.1, p<.01]$.

\section{DISCUSSION}

In recent years, there has been an explosion of interest in the finding that central directional cues trigger reflexive shifts of attention to peripheral locations (e.g.,
Driver et al., 1999; Friesen \& Kingstone, 1998; Friesen et al., 2004; Hommel et al., 2001; Langton \& Bruce, 1999; Ristic, Friesen, \& Kingstone, 2002; Ristic \& Kingstone, 2005). Originally, this work focused on the fact that reflexive orienting occurred to biologically relevant stimuli. This research usually concerned the effects of gaze direction (Driver et al., 1999; Friesen \& Kingstone, 1998; Langton \& Bruce, 1999), but it also considered the attention effects of other biologically relevant stimuli like head direction and finger pointing (see Langton et al., 2000 for a review).

Recently, research on the attention effects for directional cues has been extended to other stimuli such as arrows and words with spatial meaning (Hommel et al., 2001; Ristic et al., 2002; Tipples, 2002). Direct comparisons between these reflexive effects indicated that the behavioral effects produced by gaze and arrow cues are very similar but that the attention effect for gaze cues is more strongly reflexive than the attention effect elicited by arrow cues (Friesen et al., 2004).

The data from the present study converge onto this general result and show that, in contrast to attention effects elicited by nonpredictive eye direction, the orienting effect that is triggered by a nonpredictive arrow cue produces a performance benefit that is specific to color congruent targets. This result provides an important conceptual replication and extension to the Pratt and Hommel (2003) study, as it demonstrates that an arbitrary cue-target color contingency effect extends to the simple situation of one arrow cue presented in a single color. Importantly, the present replication occurred despite many major changes in Pratt and Hommel's methodology, including the elimination of any target-defining color precue and competing concurrent arrow-cue stimuli, suggesting that the specificity of an arrow spatial orienting effect to congruent color-targets is both robust and general.

In contrast, the orienting effect that is triggered by a spatially nonpredictive gaze cue produces a performance 
benefit that generalizes across both congruent and incongruent cue-target color contingencies. As such, this present study joins a growing list of investigations that converge on the conclusion that the attentional effects of eyes are strongly reflexive, and that as such their effect on attentional orienting appears to be resistant to changes in attentional control settings. For instance, Friesen et al. (2004) also found that participants could not help but attend first to where the eyes were directed before shifting attention to the opposite location where a target was likely. In contrast, participants were able to avoid attending to where an arrow pointed, and simply shifted their attention to the likely opposite location. A comparable resistance to change in attentional set was reported by Ristic and Kingstone (2005) who found that participants were unable to avoid orienting to where an ambiguous stimulus was gazing once they had been informed that the stimulus depicted eyes.

It is noteworthy that color congruency between gaze cues and target stimuli did affect overall RT for eyes, just as it did for arrow cues. The critical difference is that for gaze cues this factor did not interact with the significant spatial attention effect of gaze direction. Thus our observation that an interaction between cue spatial validity and cue-target color congruency does not occur for gaze cues is not merely a matter of confirming the null hypothesis. Both critical factors - reflexive orienting to gaze direction and cue-target color congruency-produced significant effects on performance. They did not, however, interact within or between gaze cue groups. These positive findings support our conclusion that the attention effect for eyes is more strongly reflexive than the attention effect for arrows; as such orienting triggered by eye direction appears to be insensitive to the effects of arbitrary cue-target color contingencies.

Like Pratt and Hommel (2003) at least some of the present results can be interpreted within the framework of attentional control setting (Folk, Remington, \& Johnston, 1992). This framework posits that the processing of a stimulus feature, such as color, is contingent on the task goal at hand, such as our participants making a response discrimination based on target color. It is possible then that the color of the cue, although it was nonpredictive, activated an attentional set for a target of a congruent color feature. In this way one can readily understand why overall RT was facilitated for targets that shared the same color as the arrow and eye cues. Conversely, violations of this attentional set may mask, or negate, any benefits of attentional orienting that is triggered by the cue in a manner that is highly reminiscent of the spotlight failure effect previously reported by Kingstone and Klein (1991; Kingstone, 1992). This interpretation reinforces the notion that attentional effects triggered by eyes are more strongly reflexive than attentional effects of arrows, insofar as the spatial orienting effect for gaze cues can withstand violations to a nonspatial color-target attentional set.

Taken together, the present data suggest that there is a range of central spatially nonpredictive stimuli that engage spatial attention reflexively, with the strength of this reflexive orienting effect varying across stimuli. Some items, like eye direction, produce strongly reflexive effects that appear to be highly resistant to violations of attentional set or modification by top-down control (e.g., Ristic \& Kingstone, 2005). Other cues, like arrows (and perhaps even to a greater extent, numbers), are sensitive to violations in attentional set and changes in top-down control settings (e.g., Galfano et al., 2006; Ristic et al., 2006). Appreciation of this point may represent an important and positive step toward the development of a coherent theory of reflexive orienting and its impact on human performance (see also Gibson \& Kingstone, 2006).

\section{AUTHOR NOTE}

This research was supported by graduate awards to J.R. from the Natural Sciences and Engineering Research Council of Canada (NSERC), the Michael Smith Foundation for Health Research (MSFHR), and the American Psychological Association; and by grants to A.K. from NSERC, the Human Frontier Science Foundation, and MSFHR. We thank Jay Pratt, Bernhard Hommel, Jennifer Stolz, and an anonymous reviewer for their comments on a previous version of this article. Correspondence concerning this article should be directed to J. Ristic, Department of Psychology, University of California, Santa Barbara, CA 931069660 (e-mail: ristic@psych.ucsb.edu) or to A. Kingstone, Department of Psychology, University of British Columbia, Vancouver, BC, V6T 1 Z4 Canada (e-mail: alan.kingstone@ubc.ca).

\section{REFERENCES}

Allison, T., Puce, A., \& McCarthy, G. (2000). Social perception from visual cues: Role of STS region. Trends in Cognitive Sciences, 4, 267278

Batki, A., Baron-Cohen, S., Wheelwright, S., Connellan, J., \& Ahluwalia, J. (2000). Is there an innate module? Evidence from human neonates. Infant Behavior \& Development, 23, 223-229.

Driver, J., Davis, G., Ricciardelli, P., Kidd, P., Maxwell, E., \& BARON-COHEN, S. (1999). Gaze perception triggers visuospatial orienting by adults in a reflexive manner. Visual Cognition, 6, 509-540.

Fischer, M. H., Castel, A. D., Dodd, M. D., \& Pratt, J. (2003). Perceiving numbers causes spatial shifts of attention. Nature Neuroscience, 6, 555-556.

Folk, C. L., Remington, R. W., \& Johnston, J. C. (1992). Involuntary covert orienting is contingent on attentional control settings. Journal of Experimental Psychology: Human Perception \& Performance, 18, 1030-1044

Friesen, C. K., \& Kingstone, A. (1998). The eyes have it! Reflexive orienting is triggered by nonpredictive gaze. Psychonomic Bulletin \& Review, 5, 490-495.

Friesen, C. K., Ristic, J., \& Kingstone, A. (2004). Attentional effects of counterpredictive gaze and arrow cues. Journal of Experimental Psychology: Human Perception \& Performance, 30, 319-329.

Galfano, G., Rusconi, E., \& Umiltà, C. (2006). Number magnitude orients attention, but not against one's will. Psychonomic Bulletin \& Review, 13, 869-874.

Gibson, B. S., \& Kingstone, A. (2006). Visual attention and the semantics of space: Beyond central and peripheral cues. Psychological Science, 17, 622-627.

Hommel, B., Pratt, J., Colzato, L., \& Godijn, R. (2001). Symbolic control of visual attention. Psychological Science, 12, 360-365.

Hood, B. M., Willen, J. D., \& Driver, J. (1998). Adult's eyes trigger shifts of visual attention in human infants. Psychological Science, 9, 131-134.

Kingstone, A. (1992). Combining expectancies. Quarterly Journal of Experimental Psychology, 44A, 69-104.

Kingstone, A., \& Klein, R. M. (1991). Combining shape and position expectancies: Hierarchical processing and selective inhibition. Journal of Experimental Psychology: Human Perception \& Performance, 17, 512-519. 
Kobayashi, H., \& Koshima S. (1997). Unique morphology of the human eye. Nature, 387, 767-768.

Langton, S. R. H., \& Bruce, V. (1999). Reflexive social orienting. Visual Cognition, 6, 541-567.

Langton, S. R. H., Watt, R. J., \& BRuce, V. (2000). Do the eyes have it? Cues to the direction of social attention. Trends in Cognitive Sciences, 4, 50-59.

Pratt, J., \& Hommel, B. (2003). Symbolic control of visual attention: The role of working memory and attentional control settings. Journal of Experimental Psychology: Human Perception \& Performance, 29, 835-845.

Ristic, J., Friesen, C. K., \& Kingstone, A. (2002). Are eyes special? It depends on how you look at it. Psychonomic Bulletin \& Review, 9, 507-513.

Ristic, J., \& Kingstone, A. (2005). Taking control of reflexive social attention. Cognition, 94, B55-B65.

Ristic, J., Wright, A., \& Kingstone, A. (2006). The number line ef- fect reflects top-down control. Psychonomic Bulletin \& Review, 13, 862-868.

Tipples, J. (2002). Eye gaze is not unique: Automatic orienting in response to noninformative arrows. Psychonomic Bulletin \& Review, 9, 314-318.

\section{NOTE}

1. The statistical equivalence between locations that sometimes receive a target (horizontal uncued) and locations that never receive a target (vertical uncued) supports the view that the spatial orienting elicited by central nonpredictive cues is reflexive. That is, the reflexive orienting effect for eyes and arrows is the same whether the cue points toward a location that may receive a target versus one that never receives a target.

(Manuscript received July 5, 2006;

revision accepted for publication January 4, 2007.) 